\title{
A Control System for Tobacco Shred Production Line Based on Industrial Ethernet
}

\author{
Li Zhang ${ }^{1}$, Guang Zheng ${ }^{1}$, Xinfeng Zhang ${ }^{2}$, Lei Liu ${ }^{2}$, and Lei Xi ${ }^{1,3, *}$ \\ ${ }^{1}$ College of Information and Management Science, Henan Agricultural University, \\ Zhengzhou, He Nan, China, 450002 \\ ${ }^{2}$ Nanyang Cigarette Factory of China Tobacco Henan Industrial Corporation, \\ Nanyang, He Nan, China, 473000 \\ ${ }^{3}$ College of Information and Management Science, Henan Agricultural University, \\ 63 Agricultural Road, Zhengzhou, 450002, P. R. China, \\ Tel.:+86-13803866921; Fax:+86-371-63558090 \\ hnaustua126.com
}

\begin{abstract}
The Industrial Ethernet based on IP realizes interconnection of industrial network and information network, and it is the most potential technology in the new industrial net products. In this paper, the defects of the original control system for tobacco shred production line are analyzed, and the new design plan of control system based on EtherNet/IP is presented. The control net adopts redundant $1000 \mathrm{M}$ fiber optic ring network that consists of six managed Industrial Ethernet Switches, and they are distributed to the central control room, leaf processing line, shred processing line, mixed stem shred processing line, online mositure regain processing line and cut tobacco dryer control cabinet. The switch in the central control room works in the pattern of redundancy management, which can switch the link in the event of the failure in link of ring net, the recovery time of link line is less than 500ms, and each main PLC of control section has dual Network Adapters. The plan has been applied for reform of $5000 \mathrm{~kg} / \mathrm{h}$ Tobacco Primary Processing Line in Nanyang Cigarette Factory of China Tobacco Henan Industrial Corporation, and the configurable software and Industry Ethernet network which has been used promots the capability of automatic control system fundamentally, showing much better transmission efficiency and reliability, realizing the goal of high cost performance and making equipment's ability of handling grow fast.
\end{abstract}

Keywords: Industrial Ethernet, tobacco shred prodution line, PLC.

\section{Introduction}

Along with the development of modern industry control technology, Industrial Ethernet network, which is easy to connect with Internet, emerging as a fast-growing unified network control technology. It has been gradually applied to the process control system (Hansen,k., 2003). The traditional industrial automatic control system

\footnotetext{
* Corresponding author.
} 
generally divided into three layers: information layer, control layer and device layer(Xia Liang, 2007). The Ethernet technology based on the TCP/IP protocol has been widely used in the modern factory information layer. Because of the higher demanding for real-time data, the application of the technology is relatively small in the control layer and device layer, especially in the device layer.

The tobacco shred production line is the first working procedure of the cigarette production, which including leaf shred production line and stem shred production line. The aims of the shred production line is to resurge, charge, cut into shred and dry the tobacco leaf and stem which has been processed in the stem-dried factory. Then the processed tobacco shreds will be blended and added fragrance in accordance with the technology requirements and finally the shredded tobacco will be produced which is suitable for future cigarette production. The stability of the shred thread production control system and its reliable operation is the key point of the tobacco shred quality insurance (Sun Xin, 2007).

The Rockwell Automation Company introduced a new industrial Ethernet technology which is based on the normative TCP/IP protocol. It integrates the normative TCP/IP protocol and CIP information protocol together. The application of this new technology into the transformation of the tobacco shred production line allows the EtherNet/IP technology applying into the control layer and the device layer. It effectively resolves the network-based control and information management of various process parameters, such as temperature and pressure of the industrial field (Yang Benyu et al., 2007). It provides a theoretical and practical basis for the information exchange and integrated automation realization among the settlement from the industrial field device layer to the control layer, management and decision layer (Zhang sheng et al., 2007).

\section{Overview of the Tobacco Shred Production Line Control System (Before Reconstruction)}

The $5000 \mathrm{KG} / \mathrm{H}$ tobacco shred production line in Nanyang Cigarette Factory of China Tobacco Henan Industrial Corporation, was put into operation in year 2002. It adopts the network structure of ControlNet and DeviceNet which is exploited by Rockwell Automation Company. This production line includes six control technology operating processing lines which are online moisture regain processing line, Leaf processing line, shred processing line, stem shred processing line, mixed stem shred processing line and flavoring processing line. Every operating stage includes a group of main control cabinet, one to two field operator stations, a distributed control box and an inverter which is distributed installed. The host controller uses ControlLogix processor and stores in the main control cabinet. The centralized control communicates though the ControlNet (fieldbus) by using RSView32 (configuration software). EtherNet standard-based C/S structure is used between monitor computer and server to finally realize the monitor of the whole production line. Network system plan before the transformation is shown in Fig.1: 


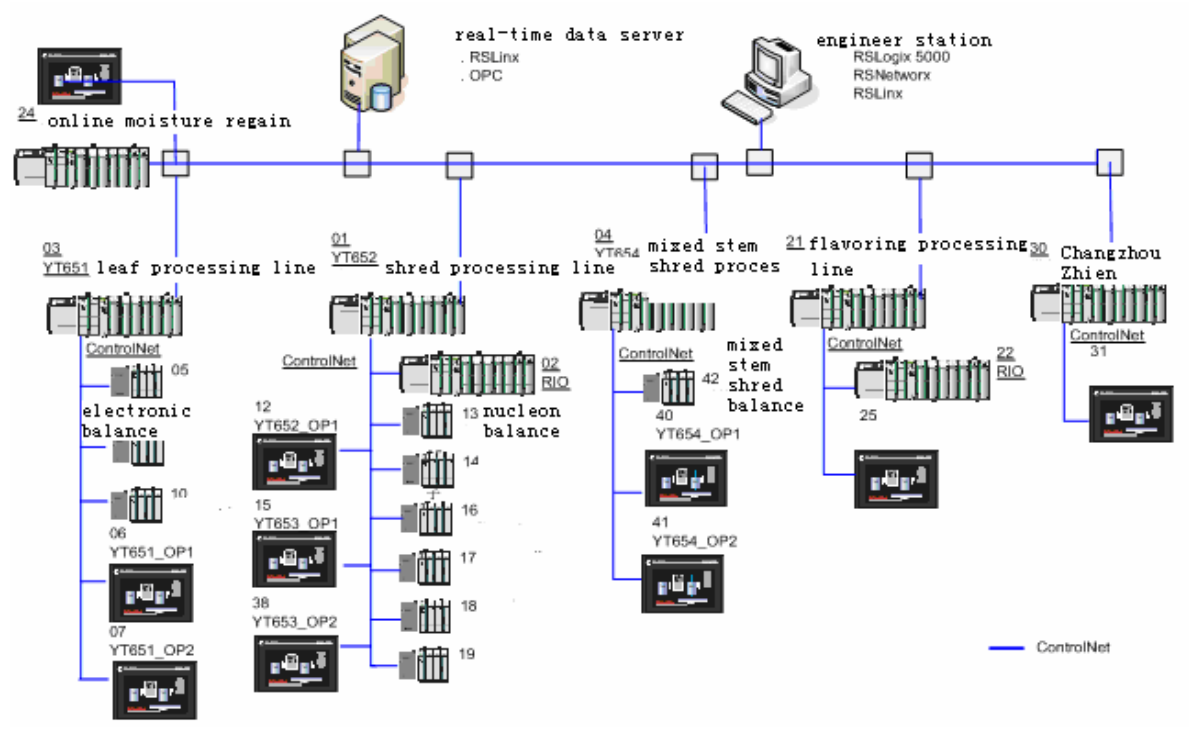

Fig. 1. The network structural plan (before reconstruction)

The problems of the system:

The network maintenance is quite difficult because it uses ControlNet network to connect all the PLC (Programmable Logic Controller) and its expansion rack together as a network. When one part of the network on PLC needs for changes (e.g. increasing templates, moving templates or increasing the expansion rack), all PLC have to stop and reorganize the network and unnecessary downtime will be happened.

Currently there are too many templates in the ControlNet Network. The scan cycle is too long which will reduce the instantaneity of the system. The communication delay and network congestion also happens sometimes.

In the central control room, all the monitors have to read PLC information though data acquisition server. When the server fails, the whole central control system cannot be used.

In the current system, the shred processing stage and stem shred processing stage together use only one CPU. As a result, the CPU will be overloaded. Moreover, in the product process, if any problems happen in one stage, the other one will also be affected.

The root cause of the above problems is using the fieldbus-based control technique. To overcome these drawbacks, the new industrial network technique-industrial Ethernet has to be used.

\section{Anylazing of the Technical Issues in the Alteration}

\subsection{Network Architecture Design}

In the early demonstration of equipment, single NIC and VLAN (Virtual LAN) technology was planning to adopt in order to improve network security and avoid 
broadcast storms. However, several problems were detected in the pilot network formation process:

VLAN settings brought about the suspension of communication across the whole network when the modules or sub-station added in the system.

Communal data transmission media made the common data channels congested.

The unclear division of authority brought about the busyness of the data source in the long term.

The system is inefficient because of two reasons: (1) there was no data sending and receiving priority; (2) different needs and different priorities could not be taken into account at the same time.

The broadcast storm, which was hoped to avoid, still came in time. It would lead to the collapse of switches easily.

Therefore, the following methods are prepared to use in the reconstruction process: 1) abolishing VLAN and using Dual NIC; 2) setting up a relatively high performance modular switches as regional automatic circuit exchange to facilitate the future expansion of facilities. Moreover, fiber link will be arranged to connect with branch switches; 3) the number of sub-stations and 1756-ENBTs (Ethernet card) should be calculated as follows: every 15 sub-stations treated as a unit, the corresponding network cards at main controller shall be increased by multiples to shunt the data streaming and improve the stability and reliability of the networks.

\subsection{Switch Selection}

Ethernet switch is one of the core equipment which plays a key role in connecting. If the switch is selected unreasonably or does not have a plenty of management features, the entire technical program would be greatly impacted. In the practical application of the process, in order to ensure industrial control, the industrial network node equipments normally issue a large number of real-time packets and a mass of network time will be used up. This phenomenon is called frequent packet in the industrial control which required lower network bandwidth and higher handling capacity of the switches. Generally, the processing capacity of the network node switches is 255 broadcast wave team groups. To meet the requirements of the network, the switches shall have management capabilities and also can discriminate every type multicast data and classify the priority of them. Otherwise, data obstruction or broadcast storms probably will happen in the network. In the alteration, fiber-optic port industrial Ethernet switches with extensive management functions will be used in the modified networks. The switch has rapid spanning tree function. Moreover, the switch in the central control room works in the redundancy management mode. Therefore, the working conditions of the ring network can be detected in the real-time. In the event of the ring link's failure, link switch can be implemented and the recovery time of the ring link would be much faster than before.

\subsection{Network Cabling}

Network cabling was another key problem in the entire engineering design. The following aspects should be fully taken into account in the initial design stage of an automated control production line: the distribution density of electrical equipment; the 
network structure and scale of the data transmission medium; the limit for data transmission; the distance and power, electromagnetic interference between the physical media; as well as the network optimization, distribution strategy and so on. Therefore, network cabling should pay attention to the following aspects: 1) the fiber-optic with good anti-interference ability should be selected between the regional switch and various branch switches. 2) Routing and connection mode which could effectively reduce the harmonic interference impact of harmonic interference must be taken for the frequency converter with larger Electro-Magnetic Interference. 3) Strong and weak electrical signal lines must be distributed separately and the professional network media need to be installed using professional equipments and tools. Professional testing tools also were required to detect the quality indicators. 4) The problem of the power interference and device node grounding, especially the problem of network grounding, must be taken into account. It is necessary to be one-point grounding for the network grounding. Multi-point grounding shall not be used in the network cabling. Otherwise, the wiring quality will be affected directly and many other problems, especially the problem of the network stability, will be found in the later stages. 5) Lightning and electrostatic protection also need be considered in the design of the machinery room environment.

\section{Modified Control System of the Tobacco Shred Production Line}

\subsection{System Structure}

The control network adopts the EtherNet/IP industrial Ethernet which developed by Rockwell Automation Company. The backbone network uses the 1000M fiber-optic ring network. The fiber-optic ring network is mainly composed by optical cables, optical connection device and industrial Ethernet switch which has network management function with extensive fiber-optic ports.

All the backbone switches are linked through multi-mode fiber and constitute a $1000 \mathrm{M}$ redundant ring network. As a communication link among monitoring layer, control layer and control device, the servers of the centralized monitoring layer and the monitors are connected to the industrial Ethernet through industrial Ethernet Switch for realizing communication, achieving data and information sharing.

Using the long-distance transmission, high anti-interference ability of fiber-optic and the rapid spanning tree function of the switches, redundant ring structure can be constituted by six managed industrial Ethernet switches. Switches are arranged in the central control room, Leaf and shred processing line, stem and shred processing line, mixed stem shred processing line, online moisture regain processing line and control cabinet of cut tobacco dryer. Among them, the switches in the central control room works in the redundancy management mode to real-time detect the working conditions of the ring network. In the event of the ring link's failure, link switch can be implemented and the recovery time of the ring network link will be less than 500 milliseconds.

The main PLC in each control stage is using dual NIC mode. One of NIC connects to the backbone network for the data communication of information layer and another NIC communicate with the underlying distributed I/O sub-station by a 100M network 
through star connection scheme. The inverter, soft starter, the smart devices instrumentation such as moisture meter and flow meter are connected by DeviceNet. The signal of the implementing agencies at the scene (e.g. motors, control valves etc.) and the detection devices (e.g. photoelectric tube, proximity switches, temperature transmitter and pressure transmitter etc.) are switching in site distributed I/O boxes. The site operator terminal and the independent control system (e.g. shred machines, electronic scales) are linked into the star network of each control stages.

Topology of the network has a good open, it links up the implementation of devices (e.g. converter, electronic scale, nuclear scale etc.), testing equipments (e.g. moisture tester of cut tobacco, infrared thermometer etc.) and automatic control equipments (tobacco cutter) from different manufacturers with Profibus. It reduced the number of modules of analog, raised speed and accuracy of data transmission, saved the Installation time, is convenient to adjusting and failure diagnostics, providing a guarantee for Precision of flow control, moisture control and temperature control. In the whole tobacco shred production line, Precision of moisture control is $1 \%$, Precision of temperature control is $2^{\circ} \mathrm{C}-3^{\circ} \mathrm{C}$, and Precision of matching is $1 \%$.

The network topology structure is shown in Fig.2.:

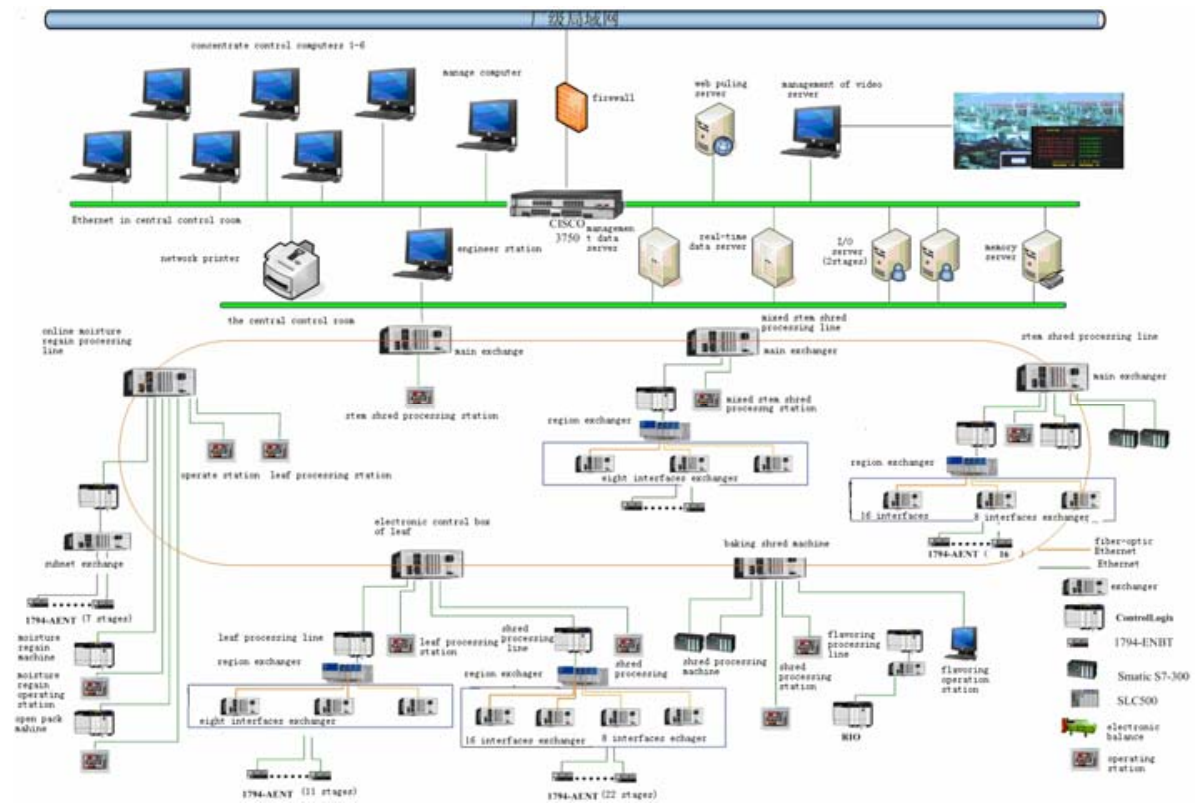

Fig. 2. The network topology structural plan.

The backbone network adopts fiber-optic ring network to connect six Hieschmann MS4128-L2PHC series of modular switches to link ControlLogix controller, two I/O server, engineer stations, real-time data server, management data server and the backup card of domain control server. The benefit of doing in this way is to reduce the number of PLC connections, lower the CPU load, and take into account for the accident which the two I/O server broken at the same time. 
In addition, there is a Cisco switch connecting the two I/O server, management data server (fault tolerance), historical database server (fault tolerance), management of application server, Web publishing server, network printer and monitors together and links to the enterprise LAN through firewall at the same time.

The branch network adapts series of modular switch and integrative switch two types switches. The ControlLogix controller with dual-card which located between the backbone network and connected to two switches separately. When one NIC or one switch is at fault, another switch or NIC can switch to the normal network in a short time to avoid data loss and inoperable condition. Upper and lower layer network both can be connected with the controller and isolated unnecessary data. As the Leaf processing line an example, the network structure is shown in Fig.3.:

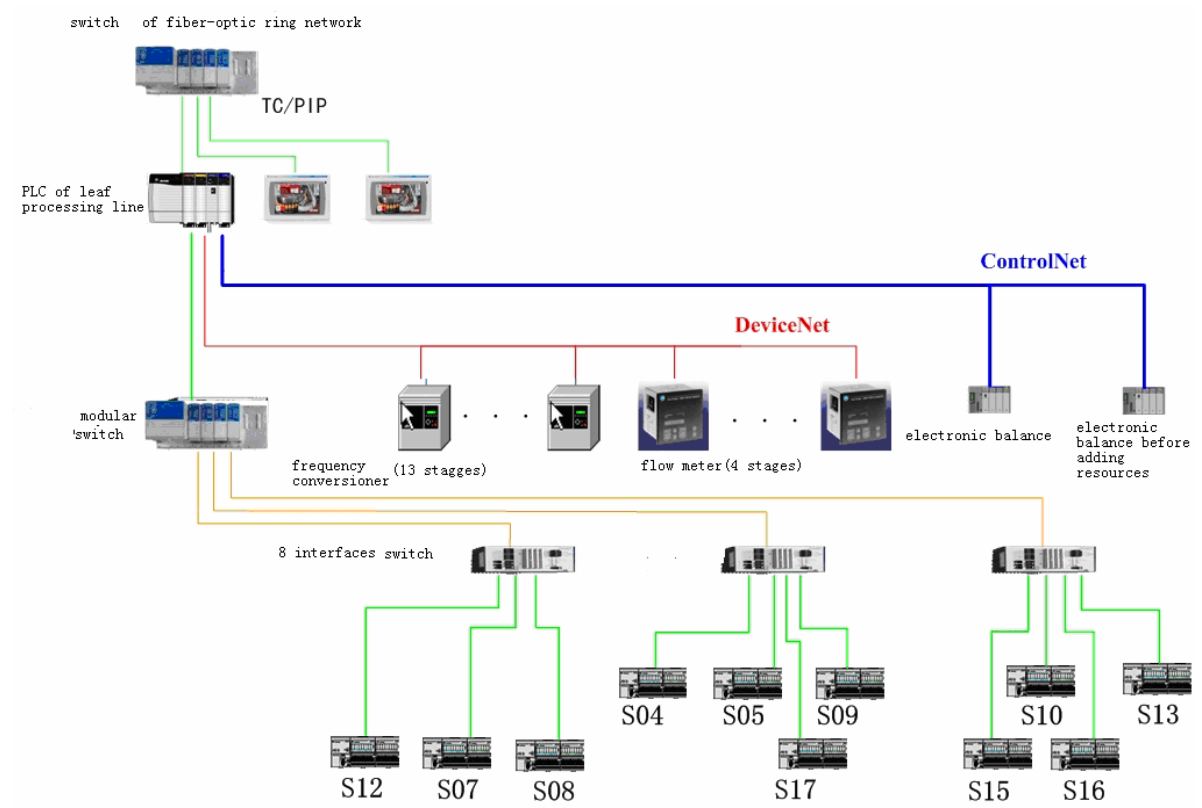

Fig. 3. The network structural plan of the leaf processing line

\subsection{Monitoring System}

The central monitor system comprises two sets of I/O server, one Oracle data server (fault tolerance), real-time database server (fault tolerance), management of application server, Web publication server and 6 monitors. It uses C/S structure to achieve monitor, data acquisition and information management functions. It sets up an engineer station to complete the programmed maintenance, system development and network maintenance. It also can communicate with other network or system though external switch and routing equipment. The field operator station uses the IFIX4.5 uniprocessor version to realize equipment monitoring. It also can solve the problem of 
different I/O servers (in the central control room) failure at the same time which would lead production suspension.

The operating system adopts Windows 2003 Server, Windows XP Professional. The monitoring software is IFIX 4.5. The communication software is RSLinx Gateway. The network software is Hirschman Hivision and the database software is Oracle 10G.

The redundant fiber optic ring network structure realizes the network redundancy and also reduces the network single point failure though a fiber -optic Ethernet ring structure. It strengthens the viability of emergency of the entire communication networks.

\section{Conclusion}

The application of the Ethernet technology in to the $5000 \mathrm{KG} / \mathrm{H}$ tobacco shred production line transformation has achieved a good effect in the Nanyang cigarette factory which affiliated to Henan tobacco industrial corporation. The production proving: the entire control system works reliably, runs in good condition, has a perfect production management function. Through the upgrading, some of the unreasonable of the original technology and design have been improved, Economic and technical efficiency are significant. The new system is also very well reviewed by users.

The application of the system configuration software and the Ethernet technology fundamentally improved the performance of the automation control system, and also show a better transmission efficiency and reliability. It realized the cost effective and achieved the goal of rapidly upgrade the equipment management and control ability. At the same time, this new network solution provides great significance for the industrial field. It provides a whole set of seamless integration system for the device layer to the layer of enterprise information system. Its technique advantage can satisfy a wide range of automation field and also can be used as an universal technique expanded to other industries, such as the electronic power, petrochemical and steel industry.

\section{References}

Crockett, N.: Industrial ethernet is ready to revolutionise the factory - Connecting the factory floor. Manufacturing Engineer, 41 (2003)

Hansen, K.: Redundancy Ethernet in industrial automation, Emerging Technologies and Factory Automation. ETFA, 943-947 (2005)

Krommenacker, N., Rondeau, E., Divoux, T.: Genetic algorithms for industrial ethernet network design. Factory Communication Systems, 149-156 (2002)

Georgoudakis, M., Kapsalis, V., Koubias, S.: Advancements, trends and real-time considerations in industrial ethernet protocols. Industrial Informatics, 112-117 (2003)

Xin, S.: Application of PLC Control in New Technology Tobacco Primary Processing Line. Electric Transmission 37(12), 56-60 (2007) (in Chinese)

Liang, X.: Implementation of comprehensive automation network system in Dafosi Mine. Coal Science and Technology 35(11), 39-42 (2007) (in Chinese) 
Benyu, Y., Weixing, Z., Donghong, W.: The design of a control system in waterworks based on configurable software and industry ethernet network. Measurement Control Technology and Instruments (9), 75-77 (2007) (in Chinese)

Xiwei, Z., Weiguo, L., Jun, K.: Study on Motor Network Control Using Industrial Ethernet. Micromotors 40(12), 59-62 (2007) (in Chinese)

Sheng, Z., Fengchun, X., Hui, C.: Supervisory system for filters in waterworks based on ControlNet. Journal of University of Shanghai For Science and Technology 29(5), 481-484 (2007) (in Chinese) 